\title{
Structure of foliations of codimension greater than one
}

Habib Marzougui and Ezzeddine Salhi

\begin{abstract}
We study the structure of a foliation of high codimension which admits a transverse foliation. We introduce four families of open saturated sets. These open sets have a simple characterization and allow us to establish a structure theorem as in codimension 1.
\end{abstract}

Mathematics Subject Classification (2000). 57R30.

Keywords. Foliation, leaves, regular open set, class, local minimal set.

\section{Introduction}

This paper concerns the study of the global structure of foliations of codimension greater than one in a closed manifold of finite dimension. In codimension 1, if the foliation is of class $C^{2}$, its topological structure is fairly well understood (cf. [2], chap.4). When the foliation is of class $C^{0}$, many phenomena occur which cannot occur in class $C^{2}$; such foliations were studied for instance in [1], [3], [4]. In codimension $q \geq 2$, there are many particular foliations which have been studied; for example, Epstein studied foliations with all leaves compact; Molino studied the Riemannian foliations case; Blummental studied the case of transversely homogeneous foliations (cf. [2], chap. 3)... It is natural to try to understand foliations in a closed manifold of codimension $q \geq 2$ : The purpose here is to develop in this general situation a setting of a structure theorem. However, there are many differences between codimension 1 and codimension $\geq 2$. But very little is known about them, in particular about their global structure. In order to generalize the case of codimension 1, we relate them to the notion of regular open set introduced by Salhi in [3], [4], and some related concepts.

We refer to section 5 for the statement of the main result of this paper.

The plan of this paper is as follows. In section 2 we give some preliminaries. In sections 3 and 4, we introduce a family of open sets and technical lemmas which will make up the main theorem. In section 5 , we prove the main theorem.

Research supported by the unit of research Algebra-Topology-Geometry: 99UR15-15. 


\section{Preliminaries}

In this section we recall some basic definitions and notations, and introduce notions which will be useful in the sequel. Let $M$ be a closed $C^{\infty}$ manifold of dimension $n$, and $\mathcal{F}$ a codimension $q, q \geq 1$ and class $C^{r}, r \geq 1$, foliation on $M$. A subset of $M$ which is a union of leaves is called an $\mathcal{F}$-saturated set. An $\mathcal{F}$-saturated set $E$ is called a local minimal set of $\mathcal{F}$ if there exists an open $\mathcal{F}$-saturated set $U$ such that $E$ is a minimal set of $\mathcal{F} \mid U$; that is for every leaf $L \subset E$, we have $\bar{L} \cap U=E$. Two distinct local minimal sets of $\mathcal{F}$ are disjoint.

We call the class of a leaf $L$ of $\mathcal{F}$ the union $\operatorname{cl}(L)$ of all leaves $G$ of $\mathcal{F}$ such that $\bar{G}=\bar{L}$. If $L$ is proper, $\operatorname{cl}(L)=L$. If $L$ is contained in a local minimal set $E$ then $\operatorname{cl}(L)=E$. An open $\mathcal{F}$-saturated set $U$ is called an open local minimal set of $\mathcal{F}$ if all leaves of $\mathcal{F} \mid U$ are dense in $U$. It is equivalent to the following: for every leaf $L$ of $\mathcal{F} \mid U, \operatorname{cl}(L)=U$. In this case, we have also $U \subset \bar{L}$.

For more information about this terminology, see [3], [4], or see also ([2], Chap. 4, section 4).

Here we give some trivial lemmas which are useful in the sequel.

Lemma 1. If $U$ is an open connected set of $M$ and $V$ is a nonempty open set of $M$ strictly contained in $U$ then $\operatorname{Fr}(V) \cap U \neq \emptyset$.

Indeed, $V$ is not closed in $U$ since $U$ is connected and distinct from $V$. Therefore $\operatorname{Fr}(V) \cap U=(\bar{V}-V) \cap U \neq \emptyset$.

Lemma 2. Let $L$ be a leaf of $\mathcal{F}$ and $X \subset \bar{L}$ be a closed nonempty $\mathcal{F}$-saturated set of $M$ with $X \neq \bar{L}$. If $U$ is the connected component of $M-X$ containing $L$ then $X=\operatorname{Fr}(U)$.

Proof. Since $U$ is closed in $M-X, \operatorname{Fr}(U)=\bar{U}-U \subset X$. On the other hand, we have $X \subset \bar{L} \subset \bar{U}$ and $X \subset M-U$. Hence $X \subset \bar{U}-U$ and then $X=\operatorname{Fr}(U)$.

The Lemma 2 is also true if we replace the leaf $L$ by any closed connected nonempty $\mathcal{F}$-saturated subset of $M$.

Corollary 1. If $G$ and $L$ are leaves of $\mathcal{F}$ such that $\bar{G} \subset \bar{L}$ and $\bar{G} \neq \bar{L}$ then $\bar{G}=\operatorname{Fr}(U)$ where $U$ is the connected component of $M-\bar{G}$ containing $L$.

In codimension $q \geq 2$, there does not necessarily exist a foliation transverse to $\mathcal{F}$ of dimension $q$. In order to give some analogies as in codimension 1 , we suppose in all the sections below, that there exists a foliation $\Gamma$ transverse to $\mathcal{F}$ of dimension $q$. 


\section{Regular and foliated fiber open sets}

In this section, we adapt to our purpose some viewpoints due to E. Salhi in [3], [4] (see also [2], chap. 4, section 4).

Let $U$ denote a nonempty open connected $\mathcal{F}$-saturated set distinct from $M$. If $T$ is a transverse leaf, we denote by $\operatorname{Sat}(T)$ its saturated set for the leaves of $\mathcal{F}$.

Definition 1. $U$ satisfies:

- Property (a): if for every $x \in U$, the transverse leaf $T_{x}$ of $\Gamma$ passing through $x$ meets the complementary $M-U$ of $U$.

- Property (c): if $U$ contains a transverse leaf and for every transverse leaf $T \subset U$, we have $\operatorname{Sat}(T)=U$.

Definition 2. $U$ is called regular if for every transverse leaf $T$ of $\Gamma$ meeting $U$, every leaf of $\mathcal{F} \mid U$ meets $T$ (i.e. $U \subset \operatorname{Sat}(T)$ ).

Definition 3. $U$ is called a foliated fiber if it is regular and satisfies Property (a).

Note that if $U=M$, we say that $M$ is regular (or a foliated fiber) if for every transverse leaf $T$ we have: $M=\operatorname{Sat}(T)$.

Remark 1. If $\mathcal{F}$ is of codimension $q=1$ :

$-U$ is a foliated fiber open set if and only if it satisfies the Property (a).

$-U$ is regular if and only if $U$ satisfies one of the Properties (a) or (c). (cf. [3], proposition 3).

Remark 2. If $\mathcal{F}$ is of codimension $q \geq 2$, a regular open set $U$ satisfies always Properties (a) or (c). The converse is not true in general:

- There exists an open set $U$ which satisfies Property (a) but it is not regular as can be shown by the following example:

Example 1. Let $\mathcal{F}=\mathcal{F}_{1} \times \mathcal{F}_{2}$ be the product foliation of $T^{3}=T^{2} \times S^{1}$ where $\mathcal{F}_{1}$ is the discrete foliation of the circle $S^{1}$ (i.e. each leaf is a single-point), and $\mathcal{F}_{2}$ is the foliation of the torus $T^{2}$ having a compact leaf $L_{0}$ and such that $\left(T^{2}-L_{0}\right)$ is a Reeb component. $\mathcal{F}$ is of codimension $q=2$. Let $\Gamma_{1}$ (resp. $\Gamma_{2}$ ) be the foliation transverse to $\mathcal{F}_{1}$ (resp. $\mathcal{F}_{2}$ ). Since $S^{1}$ is the unique transverse leaf of $\Gamma_{1}$, then any open set $U_{1} \subset S^{1}$ distinct from $S^{1}$ is a foliated fiber. Also, since $\Gamma_{2}$ has a transverse leaf $T_{2}$ such that $\operatorname{Sat}\left(T_{2}\right)=T^{2}-L_{0}$, then $T^{2}$ is not regular. It follows that the open set $U=T^{2} \times U_{1}$ in $T^{3}$ satisfies Property (a) but is not regular.

- There exists an open set $U$ which satisfies Property (c) but it is not regular as can be pointed out to us by the referee.

At first, we prepare some properties which are useful in the sequel: 
Proposition 1. Let $U_{1}$ and $U_{2}$ be open, nonempty, connected $\mathcal{F}$-saturated sets of $M$ such that $U_{1} \subset U_{2}$. Then we have the following:

(i) If $U_{2}$ is regular and $U_{1} \neq U_{2}$ then $U_{1}$ is foliated fiber.

(ii) If $U_{2}$ is an open local minimal set of $\mathcal{F}$ then $U_{1}=U_{2}$.

Proof. (i) First, $U_{1}$ is regular since it is contained in a regular set $U_{2}$. Let $T_{1}$ be a transverse leaf meeting $U_{1}$. We have $U_{2} \subset \operatorname{Sat}\left(T_{1}\right)$. Then by Lemma 1 , $\operatorname{Fr}\left(U_{1}\right) \cap \operatorname{Sat}\left(T_{1}\right) \neq \emptyset$. Therefore $T_{1}$ meets $M-U_{1}$, and $U$ satisfies Property (a).

(ii) This follows from the fact that: For every leaf $L$ of $\mathcal{F} \mid U_{2}, \operatorname{cl}(L)=U_{2}$ and then $\operatorname{cl}(L)=U_{1}$.

We introduce four families of $\mathcal{F}$-saturated open sets:

$-\mathcal{A}$ the union of all open sets which satisfy Property (a)

$-\mathcal{D}$ the union of all open local minimal sets of $\mathcal{F}$

$-\mathcal{R}$ the union of all regular open sets.

$-\mathcal{C}$ the union of all open sets which satisfy Property (c).

Note that:

$-\mathcal{A} \cap \mathcal{R}$ is the union of all foliated fiber open sets

- a regular non foliated fiber open set satisfies Property (c).

Corollary 2. Let $U$ be an $\mathcal{F}$-saturated open connected set of $M$. Then:

(i) If $U$ is an open local minimal set of $\mathcal{F}$ then $U$ is regular. In particular, we have $\mathcal{D} \subset \mathcal{R} \subset \mathcal{A} \cup \mathcal{C}$.

(ii) $U$ is an open local minimal set of $\mathcal{F}$ if and only if $U \subset \mathcal{D}$.

Proof. (i) Let $U$ be an open local minimal set of $\mathcal{F}$. If $T$ is a transverse leaf of $\Gamma$ which meets $U$ and $T_{U}$ be a connected component of $T \cap U$, then $\operatorname{Sat}\left(T_{U}\right) \subset U$ is an open, nonempty, connected $\mathcal{F}$-saturated set of $M$. It follows by (Proposition 1, (ii)) that $\operatorname{Sat}\left(T_{U}\right)=U$. Thus $U \subset \operatorname{Sat}(T)$ and $U$ is regular.

(ii) If $U$ is an open local minimal set of $\mathcal{F}$ we have $U \subset \mathcal{D}$ by definition of $\mathcal{D}$. Suppose now that $U \subset \mathcal{D}$. Since two distinct open local minimal sets of $\mathcal{F}$ are disjoint and $U$ is connected, there exists an open local minimal set $V$ of $\mathcal{F}$ such that $U \subset V$. Therefore, by (Proposition 1, (ii)) we have $U=V$.

Proposition 2. If $U_{1}$ is an open set which satisfies Property (c) and $U_{2}$ is regular such that $U_{1} \cap U_{2} \neq \emptyset$, then $U_{2} \subset U_{1}$.

Proof. By hypothesis of $U_{1}$, there exists a transverse leaf $T_{1}$ such that $\operatorname{Sat}\left(T_{1}\right)=U_{1}$. Since $U_{1} \cap U_{2} \neq \emptyset, T_{1}$ meets every leaf in $U_{1} \cap U_{2}$. Therefore $U_{2} \subset \operatorname{Sat}\left(T_{1}\right)=U_{1}$, as desired.

Corollary 3. If $U_{1}$ is an open set which satisfies Property (c) and $U_{2}$ is regular non foliated fiber such that $U_{1} \cap U_{2} \neq \emptyset$ then $U_{1}=U_{2}$. In particular, every regular non foliated fiber open set is a connected component of $\mathcal{R}$. 
Corollary 4. If $U$ satisfies Property (c) then $\operatorname{Fr}(U) \subset M-\mathcal{R}$.

Indeed, otherwise there exists a regular set $U_{2}$ such that $\operatorname{Fr}(U) \cap U_{2} \neq \emptyset$. Then $U \cap U_{2} \neq \emptyset$ and, we have $U_{2} \subset U$ (Proposition 2), a contradiction.

Proposition 3. Every connected component of $\mathcal{R}$ is regular

Proof. Suppose on the contrary, there exists a connected component $U$ of $\mathcal{R}$ which is not regular, that is, there exists a transverse leaf $T$ meeting $U$ such that $U \not \subset$ $\operatorname{Sat}(T)$. So, $U \cap \operatorname{Sat}(T)$ is nonempty and strictly contained in $U$. By Lemma 1 , $\operatorname{Fr}(\operatorname{Sat}(T)) \cap U \neq \emptyset$. Let $L$ be a leaf in $\operatorname{Fr}(\operatorname{Sat}(T)) \cap U$. There exists a regular open set $V$ such that $L \subset V$. It follows that $\operatorname{Fr}(\operatorname{Sat}(T)) \cap V \neq \emptyset$. Therefore, $V \subset \operatorname{Sat}(T)$ and then $L \subset \operatorname{Sat}(T)$, which is impossible. The proof is complete.

Corollary 5. Let $U$ be an $\mathcal{F}$-saturated open connected set of $M$. Then $U$ is regular if and only if $U \subset \mathcal{R}$.

Indeed, if $U \subset \mathcal{R}$ then there exists a regular open set $V$ such that $U \subset V$ (Proposition 3). Therefore, $U$ is regular (Proposition 1, i)).

Remark 3. If $U$ satisfies Property (a) then $U \subset \mathcal{A}$. The converse is not true in general.

\section{Technical lemmas}

Hereafter, we prepare some lemmas which play an important role in the proof of the main theorem.

Lemma 3. If $P$ is a union of transverse leaves of $\Gamma$ and $U=\operatorname{Sat}(P)$, then there exists a minimal set $E$ of $\mathcal{F} \mid U$ and $U$ is compact by saturation (i.e. quasi-compact for the topology defined by the saturated open subsets of $M$ ).

Proof. This lemma follows from Zorn's lemma since we have $\bar{P} \subset U$.

Lemma 4. Let $P_{n}(n \geq 1)$ be a union of transverse leaves of $\Gamma$. If $\operatorname{Sat}\left(P_{n+1}\right) \subset$ $\operatorname{Sat}\left(P_{n}\right)$ then $\left(\operatorname{Sat}\left(P_{n}\right)\right)_{n \geq 1}$ is stationary.

Proof. Suppose that $\left(\operatorname{Sat}\left(P_{n}\right)\right)_{n \geq 1}$ is infinite. So, we may suppose, for every $n \geq 1$ that $\operatorname{Sat}\left(P_{n+1}\right)$ is strictly contained in $\operatorname{Sat}\left(P_{n}\right)$. Let $L_{n} \subset\left(\operatorname{Sat}\left(P_{n}\right)-\operatorname{Sat}\left(P_{n+1}\right)\right)$, be a leaf of $\mathcal{F}$. Then $L_{n}$ meets a transverse leaf $T_{n} \subset P_{n}$; let $x_{n} \in T_{n} \cap L_{n}$. One can assume, passing to a subsequence if necessary, that $\left(x_{n}\right)_{n \geq 1}$ converges to a limit $x$. Let $W_{x}$ be a bidistinguished open set which contains $x$. Then, there exists an integer $N$ such that for $n \geq N, x_{n} \in W_{x}$. Therefore, the leaf $L_{n}$ of $\mathcal{F}$ 
passing through $x_{n}$ meets a transverse leaf $T_{n+1} \subset P_{n+1}$; which is impossible.

Lemma 5. Every $\mathcal{F}$-saturated open set $U$ has only a finite number of connected components which do not satisfy Property (a).

Proof. Suppose on the contrary, there exist infinitely many transverse leaves $\left(T_{n}\right)_{n \geq 1}$ in $U$ such that $\left(\operatorname{Sat}\left(T_{n}\right)\right)_{n \geq 1}$ are pairwise disjoint. Let $x_{n} \in T_{n}$ for every $n$. One can assume that $\left(x_{n}\right)_{n \geq 1}$ converges to a limit $x$. By taking a bidistinguished open set $W_{x}$ which contains $x$, there exists an integer $N$ such that for $n \geq N$, $x_{n} \in W_{x}$. Therefore, the leaf $L_{x}$ of $\mathcal{F}$ passing through $x$ meets all $T_{n}$ for $n \geq N$; a contradiction.

Lemma 6. If $\left(U_{n}\right)_{n \geq 1}$ is a sequence of open sets which do not satisfy Property (a) and with $U_{n+1} \subset U_{n}$ then there exists an open set $V \subset \underset{n \geq 1}{\cap} U_{n}$.

Proof. By assumption, for every $n$, there exists a transverse leaf $T_{n} \subset U_{n}$. Denote by $P_{n}$ the union of all transverse leaves contained in $U_{n}$, and we let $V_{n}=\operatorname{Sat}\left(P_{n}\right)$. It follows that for every $n \geq 1, V_{n+1} \subset V_{n}$ and $V_{n} \subset U_{n}$. By Lemma $4,\left(V_{n}\right)_{n \geq 1}$ is stationary; so there exists an integer $N$ such that for $n \geq N, V_{n}=V_{N} \subset U_{n}$. Hence, $V_{N} \subset \underset{n \geq 1}{\cap} U_{n}$, as desired.

Lemma 7. Let $U$ be an open set which does not satisfy Property (a). Then $U$ contains an open set which satisfies Property (c).

Proof. Suppose the contrary. Then in particular $U$ does not satisfy Property (c). There exists a transverse leaf $T_{1}$ such that $\operatorname{Sat}\left(T_{1}\right)$ is strictly contained in $U$. Since $\operatorname{Sat}\left(T_{1}\right)$ does not satisfy Property (c), there exists a transverse leaf $T_{2}$ such that $\operatorname{Sat}\left(T_{2}\right)$ is strictly contained in $\operatorname{Sat}\left(T_{1}\right)$. In this way, we construct a sequence $\left(T_{n}\right)_{n>1}$ of transverse leaves such that $\operatorname{Sat}\left(T_{n+1}\right)$ is strictly contained in $\operatorname{Sat}\left(T_{n}\right)$, which contradicts Lemma 4.

Proposition 4. If $U$ is an open set which satisfies Property (c) then $U$ has one of the following properties:

(i) $U$ is an open local minimal set of $\mathcal{F}$.

(ii) There exists a minimal set $E$ of $\mathcal{F} \mid U$ with empty interior and for every minimal set $E$ of $\mathcal{F} \mid U$, each connected component of $U-E$ satisfies Property (a).

Proof. By the hypothesis of $U$, there exists a transverse leaf $T$ of $\Gamma$ such that $\operatorname{Sat}(T)=U$. Then, by Lemma 3 there exists a minimal set $E$ of $\mathcal{F} \mid U$. Now, assume that (i) does not hold, that is $U$ is distinct from $E$, and denote by $V$ a connected component of $U-E$. We claim that $V$ satisfies Property (a). If a transverse leaf $T_{1}$ meets $V$, then one of the following holds:

(i) $T_{1}$ meets $M-U$; in this case, $T_{1}$ meets $M-V$ and $V$ satisfies Property (a). 
(ii) $T_{1}$ is contained in $U$; in this case $\operatorname{Sat}\left(T_{1}\right)=U$. Therefore, $T_{1}$ meets $M-V$, and then $V$ satisfies Property (a).

Note that a regular non foliated fiber open set satisfies Proposition 4.

Corollary 6. Let $U$ be an open set which satisfies Property (c). If $U$ contains two distinct minimal sets $E_{1}$ and $E_{2}$ then $U \subset \mathcal{A}$.

Indeed, by Proposition 4, we have $U-E_{1} \subset \mathcal{A}$ and $U-E_{2} \subset \mathcal{A}$. So, $U \subset \mathcal{A}$.

Corollary 7. If all leaves of $\mathcal{F}$ are proper then every regular non foliated fiber open set $U$ contains a leaf closed in $U$.

Proof. This follows from Proposition 4 and from the fact that a local minimal set in this case is a proper leaf.

Remark 4. If $\mathcal{F}$ is of codimension $q=1$ and $U$ satisfies one of the properties (i) or (ii) of Proposition 4 then $U$ is regular. If $\mathcal{F}$ is of codimension $q \geq 2$, and if $U$ satisfies Property (i) then $U$ is regular by Corollary 2, (i).

Proposition 5. There exist finitely many $\mathcal{F}$-saturated connected open sets $V_{1}, V_{2}$, $\ldots, V_{p}$ which satisfy Property (c) such that:

(i) $\mathcal{C}=V_{1} \cup V_{2} \cup \cdots \cup V_{p}$.

(ii) Each regular non foliated fiber open set is one of the $V_{i}, 1 \leq i \leq p$.

Proof. (i) For each $x \in \mathcal{C}$ there exists an open set $V_{x} \subset \mathcal{C}$ which satisfies Property (c). So, there exists a transverse leaf $T_{\phi(x)}$ in $V_{x}$ with $V_{x}=\operatorname{Sat}\left(T_{\phi(x)}\right)$ and $\mathcal{C}=\cup_{x \in \mathcal{C}} V_{x}$. Since $\mathcal{C}=\operatorname{Sat}\left(\cup_{x \in \mathcal{C}} T_{\phi(x)}\right)$ is compact by saturation (Lemma 3 ) then there exist finitely many transverse leaves $T_{\phi\left(x_{1}\right)}, T_{\phi\left(x_{2}\right)}, \ldots, T_{\phi\left(x_{p}\right)}$ such that $\mathcal{C}=\underset{1 \leq i \leq p}{\cup} \operatorname{Sat}\left(T_{\phi\left(x_{i}\right)}\right)=\underset{1 \leq i \leq p}{\cup} V_{x_{i}}$, as desired. Property (ii) follows from the fact that: if $R$ is a regular non foliated fiber open set then $R \cap V_{i} \neq \emptyset$ for some $i$, $1 \leq i \leq p$. It follows that $R=V_{i}$ by Corollary 3 .

\section{Main result}

Theorem 1. 1. The union $\mathcal{A} \cup \mathcal{D}$ is open and dense in $M$ and we have: $\mathcal{A} \cup \mathcal{D} \subset \mathcal{A} \cup \mathcal{R} \subset \mathcal{A} \cup \mathcal{C}$.

2. $M-\mathcal{A} \cup \mathcal{C}=\underset{1 \leq i \leq p}{\cup} \operatorname{Fr}\left(C_{i}\right)$ where $\left(C_{i}\right)_{1 \leq i \leq p}$ are the finite connected components of $\mathcal{A} \cup \mathcal{C}$ which do not satisfy Property (a).

3. $\mathcal{C}-\mathcal{A}$ is a union of finite local minimal sets of $\mathcal{F}$.

Proof. Assertion (1): Suppose the contrary; that is there exists a nonempty, open, 


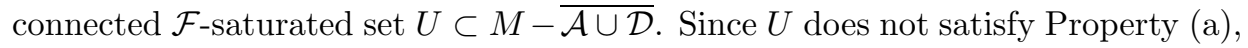
there exists by Lemma 7, a transverse leaf $T_{1}$ such that $\operatorname{Sat}\left(T_{1}\right) \subset U$ and $\operatorname{Sat}\left(T_{1}\right)$ satisfies Property (c). By Lemma 3 , there exists a minimal set $E$ in Sat $\left(T_{1}\right)$. Since $U \subset M-\mathcal{A} \cup \mathcal{D}$ we have $E \neq \operatorname{Sat}\left(T_{1}\right)$ and $\operatorname{Sat}\left(T_{1}\right)-E$ satisfies Property (a) by Proposition 4 , a contradiction.

Assertion (2): First, we deduce from assertion (1) that $\mathcal{A} \cup \mathcal{C}$ is dense in $M$. Now, let $\left(C_{i}\right)_{1 \leq i \leq p}$ be the family of connected components of $\mathcal{A} \cup \mathcal{C}$ which do not satisfy Property (a). (We know that such connected components are finite by Lemma 5). Now, since the $C_{i}$ are closed in $\mathcal{A} \cup \mathcal{C}$ then, $\underset{1 \leq i \leq p}{\operatorname{Fr}}\left(C_{i}\right) \subset M-\mathcal{A} \cup \mathcal{C}$. To prove the other inclusion $M-\mathcal{A} \cup \mathcal{C} \subset \underset{1 \leq i \leq p}{\cup} \operatorname{Fr}\left(C_{i}\right)$, let $x \in M-\mathcal{A} \cup \mathcal{C}$. Take $U_{n}=\operatorname{Sat}\left(B_{n}\right)$ where $B_{n}$ is the open ball centered at $x$ with radius $\frac{1}{n}$. Thus, $U_{n}$ is an open $\mathcal{F}$-saturated set which does not satisfy the Property (a). By Lemma 7, $U_{n}$ contains an open set $V_{n}$ which satisfies Property (c). Thus, we can suppose that for every $n, V_{n}$ is contained in one of the $\left(C_{i}\right)_{1 \leq i \leq p}$, say $C_{1}$, and $x \notin C_{1}$. Take $x_{n} \in V_{n} \cap B_{n} \subset C_{1}$. Then the sequence $\left(x_{n}\right)_{n>1}$ converges to $x$ and we have $x \in \operatorname{Fr}\left(C_{1}\right)$, this completes the proof of Assertion (2).

Assertion (3): Let $x \in \mathcal{C}-\mathcal{A}$ and $L_{x}$ be a leaf passing through $x$. From Proposition $5, x \in V_{i}$ for some $i, 1 \leq i \leq p$. We will show that $c l\left(L_{x}\right)$ is the only minimal set of $\mathcal{F} \mid V_{i}$ : If $V_{i}$ is not an open local minimal set of $\mathcal{F}$, then by Proposition 4 and Corollary 6 , there exists a unique minimal set $E_{i}$ in $V_{i}$ such that each connected component of $V_{i}-E_{i}$ satisfies Property (a). Since $x \notin \mathcal{A}$, so $x \in E_{i}$ and then $\operatorname{cl}\left(L_{x}\right)=E_{i}$.

Corollary $8([3])$. If $\mathcal{F}$ is of codimension $q=1$, then

1. $\mathcal{R}$ is open and dense in $M$.

2. There exist a finite number of half-proper leaves $L_{1}, L_{2}, \ldots, L_{p}$ of $\mathcal{F}$ such that $M-\mathcal{R}=\overline{L_{1} \cup L_{2} \cup \cdots \cup L_{p}}$

3. There exist finitely many classes which are contained in regular non foliated product sets.

Proof. Since $\mathcal{F}$ is of codimension $q=1$, we have $\mathcal{A} \cup \mathcal{C}=\mathcal{R}$. Hence, Assertion (1) follows from Theorem 1, (1). Assertion (2) follows from Theorem 1, (2) since the connected components $\left(R_{i}\right)_{1 \leq i \leq p}$ of $\mathcal{R}$ which do not satisfy Property (a) are exactly the regular non foliated product open sets and since $\operatorname{Fr}\left(R_{i}\right)$ are the closure of finitely many leaves and these leaves are half-proper and attracting in a proper side (cf. [3]). Assertion (3) follows from Theorem 1, (3).

Acknowledgements. We wish to express our thanks to the referee for reading carefully the paper and for providing us with helpful comments. 


\section{References}

[1] P. R. Dippolito, Codimension one foliations of closed manifolds, Annals of Math. 107 (1978), 403-453.

[2] C. Godbillon, Feuilletages. Études géométriques, Progress in Mathematics, Vol. 98, Birkhäuser Verlag, 1991.

[3] E. Salhi, Sur un théorème de structure des feuilletages de codimension 1, C. R. Acad. Sc. Paris, série I 300 (1985), 635-638.

[4] E. Salhi, Niveau des feuilles, C. R. Acad. Sc. Paris, série I 301 (1985), 219-222.

Habib Marzougui

Department of Mathematics

Faculty of Sciences of Bizerte

Zarzouna 7021

Tunisia

e-mail: habib.marzouki@fsb.rnu.tn
Ezzeddine Salhi

Department of Mathematics

Faculty of Sciences of Sfax

B.P. 802

3018 Sfax

Tunisia

e-mail: Ezzeddine.Salhi@fss.rnu.tn

(Received: November 30, 2000; revised version: March 20, 2001)

(17) To access this journal online:

(40) http://www.birkhauser.ch 